\title{
MULTI-SITE CHEMICAL EXCHANGE BY NMR
}

\author{
E. A. Allan, M. G. Hogben, L. W. Reevest and K. N. Shaw
}

Chemistry Department, University of Waterloo, Waterloo, Ontario, Canada

\begin{abstract}
The application of a recent multi-site chemical exchange formulation of the Bloch equations is made to an intramolecular and an intermolecular process. The experimental precautions to achieve a good complete line shape fit are discussed in some detail.

The rates of internal rotation about the $\mathrm{C}-\mathrm{N}$ bond in $\mathrm{N}, \mathrm{N}$-dimethylcarbamyl fluoride have been measured, and the relative signs of the long range couplings $\mathrm{H}-\mathrm{C}-\mathrm{N}-\mathrm{C}-\mathrm{F}{ }^{4} \mathrm{~J}$ proton to fluorine are obtained from the exchange modified spectra as well as from the comparison of the ${ }^{4} J$ measured in the fast and slow exchange limits. A 4-site rate matrix is defined with one adjustable parameter for the spectral fit. The measurements are especially difficult because of the small chemical shift varying between $2.2 \mathrm{~Hz}$ and $1.4 \mathrm{~Hz}$ over the range of interest. The long range coupling methyl to fluorine are $0.8 \pm$ $0.05 \mathrm{~Hz}$ trans and $0.3 \pm 0.05 \mathrm{~Hz}$ cis and do not vary with temperature within $0.05 \mathrm{~Hz}$ for a $16.5 \%$ solution in carbon tetrachloride.

The intermolecular exchange of halogens in the dimethyltin dihalide mixtures is formulated in detail. A 15 site exchange process breaks down into $5 \times 3$ exclusive exchange groups and providing line positions and intensities are known with the line width in the absence of exchange, the whole spectrum can be reproduced using only 2 -site rate matrix elements. Representative experimental and computed spectra are presented. The relationship of the matrix elements to chemical rate constants is derived but determinations of these constants are not yet complete.
\end{abstract}

\section{INTRODUCTION}

The study of chemical exchange rates through the modified transverse magnetization is well understood and the theory is highly developed (for example references 1-8). Some simple aspects of chemical exchange theory in $\mathrm{nmr}$ spectra for first order cases have been emphasized in a recent paper by Reeves and Shaw ${ }^{9}$. For intramolecular exchange in the first order limit, the determination of relative signs of coupling constants to non-exchanging nuclei influences the line shapes in a readily recognizable manner ${ }^{10,11}$. Exchanges among many sites (Larmor frequencies) of arbitrary population, saturation effects and site-dependent relaxation times have been accommodated in this recent theory ${ }^{9}$. A single matrix diagonalization enables sub-

†L.W.R. thanks the NRCC for financial support in the form of operating and capital equipment grants. 
sequent rapid computation of line shapes and comparison with experimental spectra ${ }^{9-11}$ to determine rate constants.

The present study is a further application to the intermolecular exchange of halogens among the dimethyltin dihalides in nonpolar solvents and an illustration of an intramolecular exchange process in $\mathrm{N}, \mathrm{N}$-dimethylcarbamyl fluoride.

Hindered rotation about the $\mathrm{C}-\mathrm{N}$ bond in amide systems has been the subject of many studies ${ }^{1,9,10,12-15}$ and there are a variety of kinetic parameters published in the literature for any given simple amide.

As an illustration of the proper application of multi-site chemical exchange theory in an experimentally difficult case, we have chosen to measure the rates of internal rotation about the $\mathrm{C}-\mathrm{N}$ bond in $\mathrm{N}, \mathrm{N}$-dimethylcarbamyl fluoride. In amides such as the fluoride, and acetamides such as the methyl and trifluoromethyl derivatives, long range coupling renders the $N$-methyl proton signals of multiplet form ${ }^{12}$. Each component of the multiplet must be treated as an individual site and magnetization transfer among the sites is restricted to pairs of components one for each of the chemically-shifted $N$-methyl signals. These individual multiplet sites are labelled by the spin states of a non-exchanging nucleus or group of nuclei to which the exchanging nuclei are coupled. This feature has generally been ignored or improperly treated ${ }^{14}$ and because of the small chemical shifts encountered in amido systems between the two groups of $N$-methyl protons a large systematic error in the rate constants results if these are treated as two simple chemically shifted sites.

Lauterbur and Burke ${ }^{16}$ in the pioneer study of ${ }^{119} \mathrm{Sn}$ chemical shifts, made an investigation of halogen exchange in mixtures of $\mathrm{SnCl}_{4}, \mathrm{SnBr}_{4}$ and $\mathrm{SnI}_{4}$. The mixed halides were formed in a moderately rapid reaction and peaks could be identified for all mixed halides $\operatorname{SnX} \mathrm{X}_{\mathbf{n}} \mathrm{Y}_{4-\mathrm{n}}$. The equilibria, as far as could be determined, represented random redistribution of halogen atoms among the tin compounds. A more recent study using proton magnetic resonance by Van der Kelen and coworkers ${ }^{17}$ of the methyltin halides showed that halogen exchange can be rapid or slow on the nmr time scale and is dependent on the particular mixed halide and the solvent in some cases. Some features of this work are difficult to explain, especially the vast change in rates of exchange between chloroform and carbon tetrachloride used as solvents. The conclusions of this paper ${ }^{17}$ were qualitative at best and in this present investigation we have concentrated on mixed dimethyltin dihalides, applied the multi-site exchange theory for quantitative measurement of rates and paid more attention to the purity of solvents.

\section{EXPERIMENTAL}

\section{$\boldsymbol{N}, \mathbf{N}$-dimethylcarbamyl fluoride (DMCF)}

Dimethylcarbamyl bromide (DMCB) was prepared by saturating $\sim 10$ $\mathrm{g}$ of dimethylcarbamyl chloride with $\mathrm{HBr}$ at $0^{\circ} \mathrm{C}^{18}$. The DMCF was then prepared by an exchange reaction between equimolar amounts of DMCB and silver fluoride in acetonitrile. A more efficient reaction was achieved in 
a second preparation by using $\mathrm{SbF}_{3}$ as a fluorinating agent ${ }^{18}$. The DMCF was purified by a spinning band column distillation at $760 \mathrm{mmHg}$ and $121^{\circ} \mathrm{C}$. Elemental analysis gave $\mathrm{C}=39.81(39.58) \mathrm{H}=6.58(6.59) \mathrm{F}=20.68(20.88)$ the values in brackets being theoretical for $\left(\mathrm{CH}_{3}\right)_{2} \mathrm{NCOF}$.

\section{Dimethyltin dihalides}

Dimethyltin dichloride and dimethyltin dibromide were obtained commercially and purified by subliming in vacuo at room temperature. Dimethyltin diiodide was prepared in the laboratory by heating appropriate molar proportions of tetramethyltin and tin tetraiodide (Alpha Inorganic) in a sealed tube for 2-3 days at temperatures from $150^{\circ}$ to $225^{\circ} \mathrm{C}$ with frequent agitation. Dimethyltin diiodide was separated by distillation at reduced pressure from the other methyltin iodides which formed. Dimethyltin diiodide is a solid at room temperature and was easily separated from liquid trimethyltin iodide which drained from the water-cooled condenser. Extraction of the crude product with chloroform was followed by recrystallization and further sublimation under reduced pressure. The purified sample melted at $40^{\circ} \mathrm{C}$. In all cases $\mathrm{nmr}$ spectra showed no impurities under the highest gain conditions of the HA-100 spectrometer when samples were examined in solvents.

For the chemical exchange studies samples of dihalide were weighed into $5 \mathrm{~mm}$ OD $\mathrm{nmr}$ tubes and solvent added to the required volume. The chemical exchange rates of the halogens in these systems are extremely sensitive to water impurity or hydroxylic solvents. In order to retain the same line shape indicating unchanged exchange rates over periods of several months, it was found convenient to insert a glass wool plug in the upper part of the $\mathrm{nmr}$ tube and suspend above this some molecular sieve (Linde $4 \mathrm{~A}^{\circ}$ ). After sealing the tube following several freeze pump thaw cycles, the inversion of the tube ensured storage of the solution over an intense drying agent.

\section{NMR measurements}

Proton magnetic resonance measurements were obtained using a Varian HA-100 spectrometer equipped with a V-6031 variable temperature probe and temperature controller. Temperature measurements in the $\mathrm{nmr}$ probe were made by using the relative chemical shifts of a methanol- $\mathrm{OH}$ or glycol - $\mathrm{OH}$ proton in the appropriate temperature region. Temperature measurements were made before and after every spectrum. The interpolation of the - OH chemical shifts was taken from the data of Van Geet ${ }^{19}$. The temperature measurement and control at the sample was $\pm 0.3^{\circ} \mathrm{C}$ maximum error at the extremes of the highest and lowest temperatures. Small temperature gradients do occur along the approximately $2 \mathrm{~cm}$ length of the sample. These do not however influence the spectra a great deal. The majority of the proton signal arises from sample within one coil diameter $(\sim 7 \mathrm{~mm})$ up and down the sample from the centre of the receiver coil and the temperature gradient effect is thereby minimized A total variation of approximately $1.5^{\circ} \mathrm{C}$ can be observed from the top to the bottom of the $2 \mathrm{~cm}$ liquid sample when the mean temperature differs approximately $100^{\circ} \mathrm{C}$ from ambient, otherwise closer to room temperature the effect is much less. A temperature gradient always leads to a broader line which overestimates the rate constant 
in the slow exchange region and underestimates it in the fast exchange region. The fitting to a Lorentzian form in the appropriate region leads to maximum errors in the frequency range 2 to 5 line widths if there is a temperature gradient. Base line errors are equaliy serious in this region so that we conclude the conditions of our temperature measurement and control is not a serious systematic error. The temperature control sensor causes the intermittent use of a heater in the nitrogen gas stream. Cycling of the temperature due to this heater does occur and can be measured by very careful chemical shift observations on the methanol- $\mathrm{OH}$ peak as a function of time. The nitrogen gas flow rate can be optimized at each temperature so as to minimize the cycling effect and reduce it to values negligible compared to the error inherent in the temperature gradient. The matter of errors due to temperature measurement and control have been largely neglected in kinetic studies by $\mathrm{nmr}$, but we expect to treat this part of the problem more extensively in a future paper.

At least four spectra at any temperature were stored in a Fabritek 1074 multi-channel analyzer interfaced to the HA-100. At least 50 and often more than 200 intensity points were digitized conveniently from the experimental spectra in this manner. It is important to continuously monitor the line width and shape in the absence of a chemical exchange effect at all times. This is most conveniently achieved by choosing a compound with a single sharp proton resonance not undergoing exchange which has a chemical shift near the exchanging protons but not so close as to interfere with estimates of base line. The proton resonance signal of this compound was optimized to minimum line width and Lorentzian shape by adjustment of the homogeneity controls of the spectrometer, at all temperatures. It is usually included in the sample to the extent of $2 \%$ and will be referred to in the text as the line shape standard. The line width was always maintained better than $0.3 \mathrm{~Hz}$ and sometimes it could be reduced to $0.15 \mathrm{~Hz}$. For the studies on DMCF, dioxane provided an excellent line shape standard. Wilmad PS-505 $5 \mathrm{~mm}$ thin-walled sample tubes were utilized and found to give excellent mechanical spinning characteristics and good reproducibility of field homogeneity control. Spectra were recorded at sweep rates of 0.02 or $0.05 \mathrm{~Hz} \mathrm{~s}^{-1}$ in the approximately slow passage limit ${ }^{20}$ with an $H_{1}$ field $0.02 \mathrm{mG}$, such conditions being well below the requirements for a non-saturated line ${ }^{20}$.

Intensity measurements required for the study of equilibrium quotients in the methyltin halide systems, were made by repeated tracings (at least five per spectrum) onto good quality bond paper and cutting out the spectra for weighing of individual peaks. Tetramethylsilane or hexamethyl disiloxane was added to the extent of $1 \%$ in almost all samples to provide a suitable sharp peak for a field-frequency lock.

\section{THEORY}

\section{Computation of line shapes}

In a simplified matrix formulation of the Bloch equations ${ }^{2}$ modified for chemical exchange, Reeves and Shaw ${ }^{9}$ obtained the expression below for the transverse magnetization for $n$ Larmor frequencies, in the absence of saturation: 


$$
G(x)=-i \omega_{1} M_{0} \text { I.S. }[\boldsymbol{\Lambda}+i x \boldsymbol{I}]^{-1} \cdot S^{-1} . P
$$

where $S$ is the matrix which diagonalizes $\boldsymbol{R}$, a modified rate of exchange matrix

$$
\boldsymbol{\Lambda}=\boldsymbol{S}^{-1} \cdot \boldsymbol{R} . \boldsymbol{S}
$$

Once the matrix $S$ has been defined, the extraction of the imaginary part ${ }^{2}$ of $G(x)$ is computationally greatly simplified. Since $\boldsymbol{A}$ is diagonal and $\boldsymbol{I}$ is the unit matrix then the inversion $[\boldsymbol{\Lambda}+i x \boldsymbol{I}]^{-1}$ is trivial for each data point $x$. $x$ is a frequency difference $\left(\omega-\omega_{0}\right)$ from some arbitrary reference frequency in the spectrum, $\omega_{0}$. A diagonal $n \times n$ chemical shift matrix $\Omega$ is included in $\boldsymbol{R}$ and merely defines line positions $\Omega_{i}=\omega_{i}-\omega_{0}$ for the $n$ Larmor frequencies. The term chemical shift matrix is somewhat misleading in the sense that individual Larmor frequencies may arise from first order multiplets caused by spin-spin coupling. The theory is limited to the first order case i.e. $J \ll \delta$ for all chemically shifted peaks in the spectrum. A computer program GPLONK has been written to accommodate exchange of transverse magnetization among up to 24 sites and is iterative to optimize the fit of the complete line shape. The various stages of iteration can be controlled to optimize first the site exchange rates, the line positions and finally the apparent line widths in the absence of any exchange process.

The input data requires the definition of the following matrices and vectors :

(a) The chemical shift matrix $\Omega$.

Diagonal with elements $\Omega_{i}=\left(\omega_{i}-\omega_{0}\right)$

These are obtained generally to a first approximation by observation in the slow exchange limit and any variation with temperature can be estimated in the slow exchange region to be extrapolated as first order estimates in the fast exchange region. In general, any variations of $\Omega_{i}$ s with temperature, were found to be linear. In minimizing the mean error per data point a final adjustment of line positions is very important and will be described in the results section.

(b) The population vector $\mathbf{P}$.

For the purposes of normalizing intensities and taking account of concentrations, a column vector $\mathbf{P}$ defines the fractional populations $P_{i}$ in the $n$ sites, undergoing exchange of transverse magnetization. These may be temperature dependent.

(c) The rate matrix $\boldsymbol{R}_{2}$.

$$
\boldsymbol{R}_{2}=\boldsymbol{T}_{2}+\boldsymbol{K}
$$

$T_{2}$ is a diagonal matrix $n \times n$ with elements $T_{2}^{*-1}$. In an ideal spectrometer of infinite resolution the values $T_{2_{i}}$ are site dependent. The resolution limitation of the magnet where $T_{2_{i}}^{*} \ll T_{2_{i}}$ for all sites, means that the diagonal matrix $\boldsymbol{T}_{2}$ has equal diagonal elements defined by the line shape standard. In no case discussed in this paper is this statement not true. $\boldsymbol{K}$ is a site rate matrix $n \times n$.

The diagonal elements are given by

$$
K_{i i}=\sum_{\substack{j=1 \\ j \neq i}}^{n} k_{i j}
$$


$k_{i j}$ are pseudo-first order rate constants for the transfer of transverse magnetization from site $i$ to all other sites $j$. In general this pseudo-first order data constant has $(n-1)$ components to the other $(n-1)$ sites. The off-diagonal elements are given by

$$
K_{i j}=-k_{j i}, j \neq i
$$

These elements represent the individual pseudo-first order rate constants for magnetization transfer from site $j$ back to site $i$. The detailed balancing of rates for site $i$ at equilibrium require

$$
K_{i i}+\sum_{j \neq i}^{n} K_{j i}=0
$$

i.e. the sum for each column is zero. It is clear that in this context the word 'site' is synonymous with 'individual Larmor frequency'. Translation into chemical terms in the case of intermolecular processes will be discussed later.

\section{Intramolecular exchange}

In an intramolecular exchange process such as the exchange of two methyl groups in amido-type systems by rotation about the $\mathrm{C}-\mathrm{N}$ bond, any spinspin coupling to a non-exchanging nucleus increases the number of Larmor frequencies and places restrictions on the number of site exchanges which can occur $^{21}$. This has implications with regard to the determination of relative signs of spin-spin coupling constants through the chemical exchange process $^{10,13}$. In general no spin-spin coupling is observed between the two sets of methyl group protons in amido systems (exception is the carbamyl cyanide). The spectra observed are often of the type $\mathrm{A}_{3} \mathrm{~B}_{3} \mathrm{X}_{\mathrm{n}}$ with $J_{\mathrm{AB}}=0$. As an example of the relative signs of coupling influencing the spectrum, when protons $\mathrm{A}$ and $\mathrm{B}$ are exchanging, the spectrum $\mathrm{ABX} J_{\mathrm{AB}}=0$ contains all the features necessary.

In Figure 1 a line position diagram is given for the $\mathrm{AB}$ part of this $\mathrm{ABX}$ case. In the upper part (a) the four lines are labelled according to the chemical shift A or B and the spin state of the $X$ nucleus to which they are coupled. The diagram Figure 1 (a) corresponds to the same sign for $J_{\mathbf{A X}}$ and $J_{\mathbf{B X}}$. The spin state lifetime of the $\mathrm{X}$ nucleus is long compared to the exchange lifetime under the conditions where lines are significantly broadened. In preserving the integrity of the $\mathrm{X}$ spin state, exchange occurs between lines 1 and 3 and 2 and 4. In the computation the rate matrix becomes

$$
\left[\begin{array}{rrrr}
k & 0 & -k & 0 \\
0 & k & 0 & -k \\
-k & 0 & k & 0 \\
0 & -k & 0 & k
\end{array}\right]
$$

where $k$ is the rate constant for interchange of A and B chemically shifted sites. In the limit of extreme fast exchange the two remaining lines are separated by $J+=\frac{1}{2}\left(J_{\mathrm{AX}}+J_{\mathrm{BX}}\right)$. In Figure 1 (b) the spin states of nucleus $\mathrm{X}$ are labelled for opposite relative signs $J_{\mathrm{AX}}, J_{\mathrm{BX}}$. Again preserving the $I_{z}$ value of the $\mathrm{X}$ nucleus during the exchange the matrix $k$ becomes : 
MULTI-SITE CHEMICAL EXCHANGE BY NMR

$$
\left[\begin{array}{rrrr}
k & 0 & 0 & -k \\
0 & k & -k & 0 \\
0 & -l & k & 0 \\
-k & 0 & 0 & k
\end{array}\right]
$$

In the limit of fast exchange the two line averaged $\mathrm{AB}$ spectrum has separation $\frac{1}{2}\left(J_{\mathrm{BX}}-J_{\mathrm{AX}}\right)$ if $J_{\mathrm{AX}}<J_{\mathbf{B X}}$. The only change in the computation is the rate matrix; line positions and intensities remain the same. The line shapes at all rates of exchange between slow and fast limits are quite different for the same

(a)
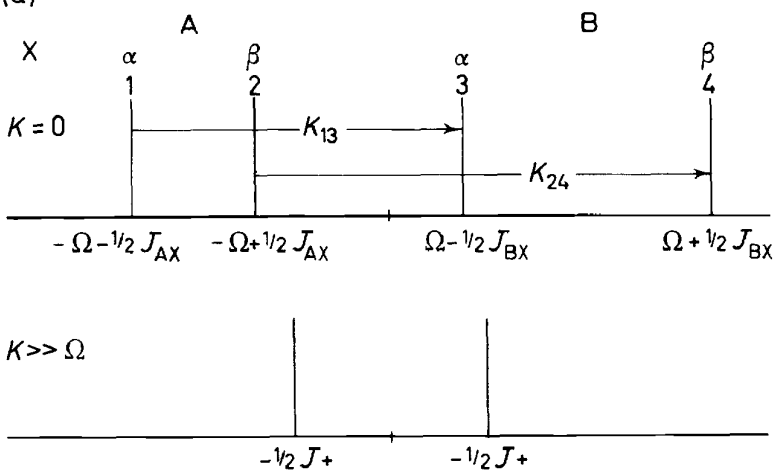

(b)
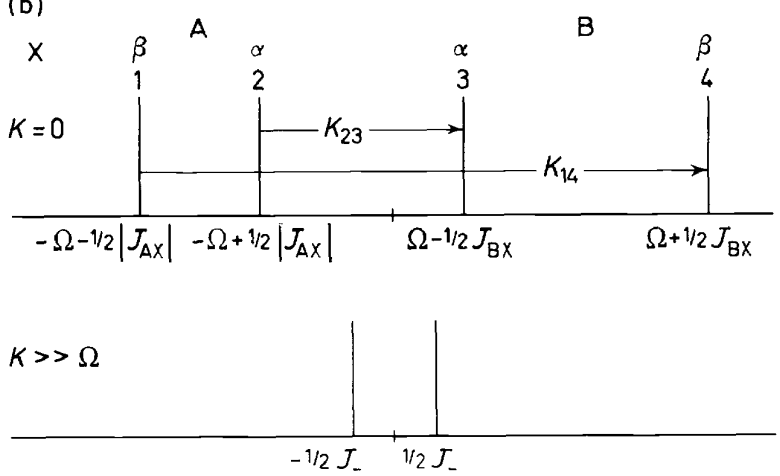

Figure 1. Intramolecular chemical exchange for an $\mathrm{ABX}$ spectrum with $J_{\mathrm{AB}}=0, J_{\mathrm{AX}}, J_{\mathrm{BX}} \neq 0$, (AB part). The four transition frequencies are shown diagrammatically labelled above each transition with the appropriate spin state of the $X$ nucleus. In part (a) above, the spin states of $X$ are labelled for like signs $J_{\mathrm{AX}}$ and $J_{\mathrm{BX}}$. In the lower part (b) of the figure, spin states of $\mathrm{X}$ are labelled for opposite relative signs $J_{\mathrm{AX}}, J_{\mathrm{BX}}$. The horizontal lines in the slow exchange limits $K=0$ correspond to the possible transverse magnetization exchanges in each case.

and opposite relative signs. This has been applied in the $\mathrm{A}_{3} \mathrm{~B}_{3} \mathrm{X}$ spectrum of $N, N$-dimethylcarbamyl fluoride to determine relative signs of $J_{\mathrm{AX}}$ and $J_{\mathrm{BX}}$. The $A_{3} B_{3}$ part of the spectrum can be treated exactly as above. 


\section{Intermolecular exchange}

The same first order chemical exchange theory can be applied, provided $J \ll \delta$ for all exchanging sites. Interruption of spin coupling by chemical exchange can be included with no extra difficulty. The case of the application of theory for exchange of halogens among the dimethyltin dihalides will be specifically discussed here. A chemically sensible kinetic scheme is first set up for the intermolecular exchange mechanism. In the case of the exchange of halogens among two dimethyltin dihalides the reactions can be complex. The chemical equilibrium

$$
\left(\mathrm{CH}_{3}\right)_{2} \mathrm{SnX}_{2}+\left(\mathrm{CH}_{3}\right)_{2} \mathrm{SnY}_{2} \underset{k_{-1}}{\stackrel{k_{1}}{=}} 2\left(\mathrm{CH}_{3}\right)_{2} \mathrm{SnXY}
$$

represents two bimolecular reactions with chemical rate constants $k_{1}$ and $k_{-1}$.

The following halogen exchange reactions may simultaneously occur:

$$
\begin{aligned}
& \left(\mathrm{CH}_{3}\right)_{2} \mathrm{Sn}^{\dagger} \mathrm{X}_{2}+\left(\mathrm{CH}_{3}\right)_{2} \mathrm{SnXY} \stackrel{k_{2}}{\rightarrow}\left(\mathrm{CH}_{3}\right)_{2} \mathrm{Sn}^{\dagger} \mathrm{XY}+\left(\mathrm{CH}_{3}\right)_{2} \mathrm{SnX}_{2} \\
& \left(\mathrm{CH}_{3}\right)_{2} \mathrm{Sn}^{\dagger} \mathrm{Y}_{2}+\left(\mathrm{CH}_{3}\right)_{2} \mathrm{SnXY} \stackrel{k_{3}}{\rightarrow}\left(\mathrm{CH}_{3}\right)_{2} \mathrm{Sn}^{\dagger} \mathrm{XY}+\left(\mathrm{CH}_{3}\right)_{2} \mathrm{SnY}_{2}
\end{aligned}
$$

The reactions 8 and 9 represent unsymmetric exchange of halogen between both dihalides and the mixed halide.

The following reactions may also take place at the same time:

$$
\begin{aligned}
& \left(\mathrm{CH}_{3}\right)_{2} \mathrm{Sn}^{\dagger} \mathrm{XX}^{*}+\left(\mathrm{CH}_{3}\right)_{2} \mathrm{SnX}_{2} \stackrel{k_{4}}{\rightarrow}\left(\mathrm{CH}_{3}\right)_{2} \mathrm{Sn}^{\dagger} \mathrm{X}_{2}+\left(\mathrm{CH}_{3}\right)_{2} \mathrm{SnXX} X^{*} \\
& \left(\mathrm{CH}_{3}\right)_{2} \mathrm{Sn}^{\dagger} \mathrm{YY}^{*}+\left(\mathrm{CH}_{3}\right)_{2} \mathrm{SnY}_{2} \stackrel{\mathrm{ks}}{\rightarrow}\left(\mathrm{CH}_{3}\right)_{2} \mathrm{Sn}^{\dagger} \mathrm{Y}_{2}+\left(\mathrm{CH}_{3}\right)_{2} \mathrm{SnYY} \mathrm{Y}^{*} \\
& \left(\mathrm{CH}_{3}\right)_{2} \mathrm{Sn}^{\dagger} \mathrm{XX}^{*}+\left(\mathrm{CH}_{3}\right)_{2} \mathrm{SnXY} \stackrel{k_{6}}{\rightarrow}\left(\mathrm{CH}_{3}\right)_{2} \mathrm{Sn}^{\dagger} \mathrm{X}_{2}+\left(\mathrm{CH}_{3}\right)_{2} \mathrm{SnX}{ }^{*} \mathrm{Y} \\
& \left(\mathrm{CH}_{3}\right)_{2} \mathrm{Sn}^{\dagger} \mathrm{YY}^{*}+\left(\mathrm{CH}_{3}\right)_{2} \mathrm{SnXY} \stackrel{k_{7}}{\rightarrow}\left(\mathrm{CH}_{3}\right)_{2} \mathrm{Sn}^{\dagger} \mathrm{Y}_{2}+\left(\mathrm{CH}_{3}\right)_{2} \mathrm{SnXY} \mathrm{X}^{*} \\
& \left(\mathrm{CH}_{3}\right)_{2} \mathrm{Sn}^{\dagger} \mathrm{X}^{*} \mathrm{Y}+\left(\mathrm{CH}_{3}\right)_{2} \mathrm{SnXY} \stackrel{k_{3}}{\rightarrow}\left(\mathrm{CH}_{3}\right)_{2} \mathrm{Sn}^{\dagger} \mathrm{XY}+\left(\mathrm{CH}_{3}\right)_{2} \mathrm{SnX} \mathrm{X}^{*} \mathrm{Y} \\
& \left(\mathrm{CH}_{3}\right)_{2} \mathrm{Sn}^{\dagger} \mathrm{XY}^{*}+\left(\mathrm{CH}_{3}\right)_{2} \mathrm{SnXY} \stackrel{k_{9}}{\rightarrow}\left(\mathrm{CH}_{3}\right)_{2} \mathrm{Sn}^{\dagger} \mathrm{XY}+\left(\mathrm{CH}_{3}\right)_{2} \mathrm{SnXY}^{*}
\end{aligned}
$$

All the reactions represented by equations $10-15$ are symmetric in the halogen exchanged and no modulation of the methyl proton resonance signal occurs. The line shapes of experimental spectra derive from reactions with bimolecular rate constants $k_{1}, k_{-1}, k_{2}$ and $k_{3}$. Naturally-occurring tin has two magnetic isotopes with spin half properties ${ }^{117} \mathrm{Sn}$ and ${ }^{119} \mathrm{Sn}$; these have abundances $7.67 \%$ and $8.68 \%$. Tin satellites are observed on each side of the methyl proton resonances, and being of similar gyromagnetic ratios the two sets of satellites appear close together. The total spectrum in the slow exchange limit consists of 15 peaks. Chemical exchange of the type outlined in equations 7-9 does not involve breaking of the $\mathrm{Sn}-\mathrm{C}$ bond so that the spin-spin splitting is preserved during the exchange. This has important consequences on the Larmor frequencies which are modulated by the exchange. In Figure 2 a diagrammatic representation of the 15-line spectrum is given. The exchange processes modulate frequencies between the three main peaks $\left(\mathrm{CH}_{3}\right) \mathrm{SnX} \mathrm{X}_{2},\left(\mathrm{CH}_{3}\right)_{2} \mathrm{SnXY}$ and $\left(\mathrm{CH}_{3}\right)_{2} \mathrm{SnY}_{2}$ and between corresponding tin satellites. As it is highly unlikely that the tin-proton coupling constants change sign for the three molecules above, the exchange processes modulate low field tin satellites and high field tin satellites separately. Indeed this is in principle a determination of the relative signs of spin-spin coupling constants 
MULTI-SITE CHEMICAL EXCHANGE BY NMR

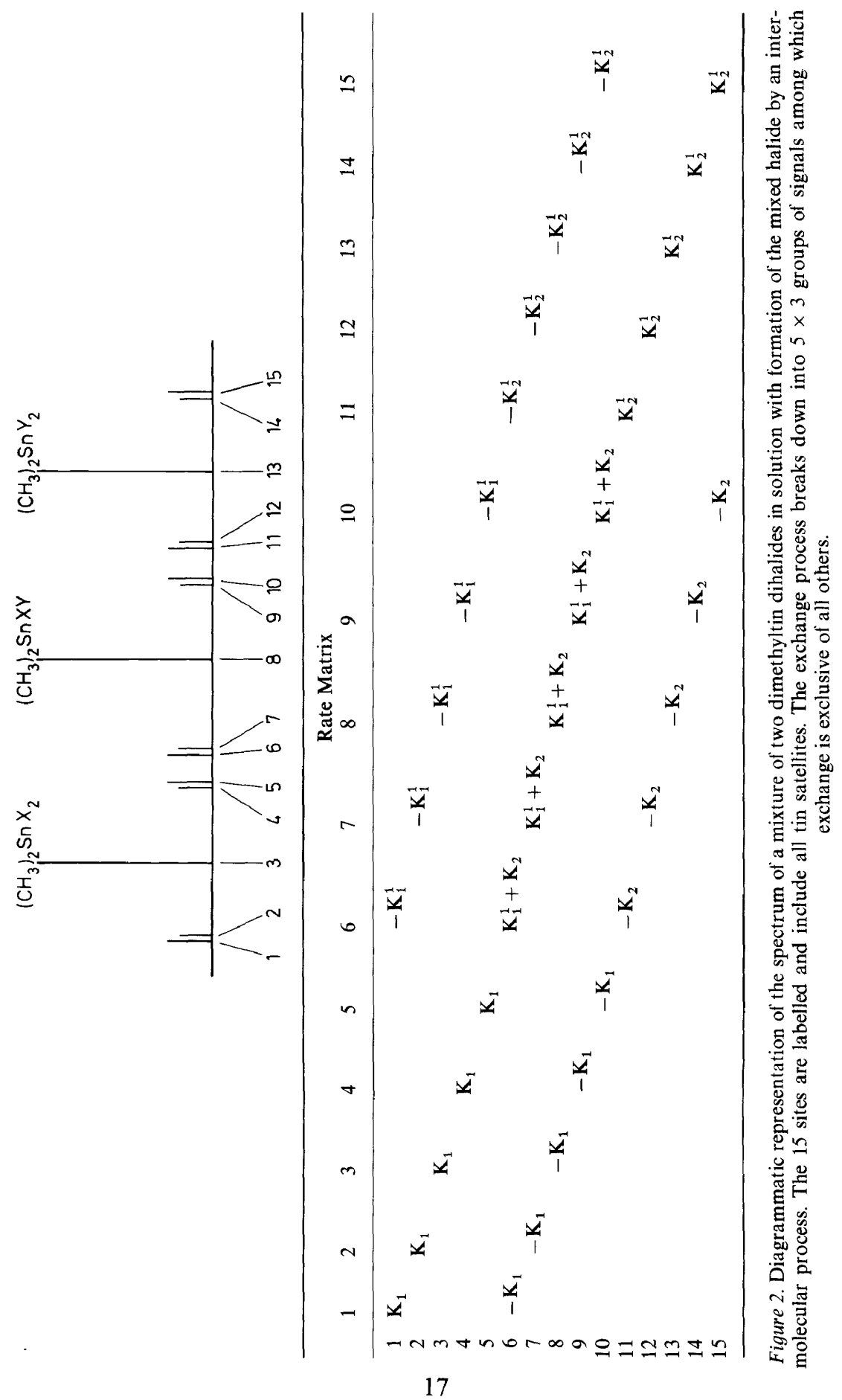


in an intermolecular exchange. It is possible that cases exist where one molecule converts into another and there is a change in the sign of the coupling. The rate matrix presented in Figure 2 is appropriate for the same relative signs of the tin-proton coupling and connects all Larmor frequencies $i j$ which exchange magnetization. There are only two independent 'site rate matrix' eiements. As an example the three principal peaks 3,18 and 13 of Figure 2 can be treated.

The pseudo-first order rate constants are

$$
\begin{aligned}
& K_{8,3}=k_{1}\left[\mathrm{SnY}_{2}\right]+k_{2}[\mathrm{SnXY}] \\
& K_{3,8}=k_{-1}[\mathrm{SnXY}]+k_{2}\left[\mathrm{SnX} X_{2}\right] \\
& K_{13,8}=k_{3}\left[\mathrm{SnY}_{2}\right]+k_{-1}[\mathrm{SnXY}] \\
& K_{8,13}=k_{1}\left[\mathrm{SnX}_{2}\right]+k_{3}[\mathrm{SnXY}]
\end{aligned}
$$

Detailed balancing of the rates for two sites gives the following equalities

$$
\begin{aligned}
K_{3,8} p_{8} & =K_{8,3} p_{3} & & \text { Site } 3 \\
K_{8,13} p_{13} & =K_{13,8} p_{8} & & \text { Site } 13
\end{aligned}
$$

there are thus only two independent site rate matrix elements for the three sites, which can be designated $K_{1}=K_{3,8}$ and $K_{2}=K_{8,13}$. Since all exchanges are connected by the same chemical reactions, three peaks at a time $-1,6$ and $11 ; 2,7$ and $12 ; 4,9$ and 14 ; and 5,10 and 15 ; then the rate matrix has only two independent elements.

The population vector takes into account the intensities of each peak, with the total intensity normalized to one and this implies a knowledge of the equilibrium quotient

$$
K_{Q}=\frac{[\mathrm{SnXY}]^{2}}{\left[\mathrm{SnY}_{2}\right]\left[\mathrm{SnX}_{2}\right]}=\frac{k_{1}}{k_{-1}}
$$

The experimental line shape can be fitted by use of $K_{1}$ and $K_{2}$ with a population vector, the frequencies of the peak positions and a diagonal relaxation matrix $\boldsymbol{T}_{2}$.

There are four unknown chemical rate constants $k_{1}, k_{-1}, k_{2}$ and $k_{3}$ in equations 16 and 18 and three known concentrations. A knowledge of the equilibrium quotient can be used if it is satisfactorily a constant over the concentrations of interest. The value of $K_{Q}$ then gives a ratio of two chemical rate constants as in equation 21 . By changing the known concentrations two new matrix elements $K_{3}$ and $K_{4}$ can be determined and this is sufficient to determine the chemical rate constants, with or without the equilibrium quotient. In practice, in order to overdetermine the system, several concentrations are used to minimize the errors in individual measurements.

\section{Analysis of fitting errors}

The total line shape fitting errors are expressed in terms of the mean error per data point

$$
\varepsilon=\frac{1}{N} \sum_{i=1}^{N}\left[V^{1}\left(x_{i}\right)-V\left(x_{i}\right)\right] / V\left(x_{i}\right)
$$




\section{MULTI-SITE CHEMICAL EXCHANGE BY NMR}

where $V^{1}\left(x_{i}\right)$ and $V\left(x_{i}\right)$ are the normalized experimental and theoretical intensities, respectively for the frequency $x_{i} . N$ is the total number of data points across the spectrum. Usually this error can be less than $2 \%$ except for the extreme fast and slow exchange regions where determination to $6 \%$ deviation is acceptable.

\section{RESULTS}

\section{Internal rotation rates about the $\mathbf{C}-\mathrm{N}$ bond in DMCF}

Line shape measurements were made on a degassed sample 16.5 mole \% $\mathrm{DMCF}$ in carbon tetrachloride. The sealed tube was filled with nitrogen gas at one atmosphere to prevent boiling at too low a temperature in the fast exchange region. A spectrum of the protons in DMCF taken at $220 \mathrm{MHz}$ gave a chemical shift measurement between the two methyl protons groups as $3.6 \pm 0.1 \mathrm{~Hz}$ for a $7.5 \%$ solution in carbon tetrachloride at $22^{\circ} \mathrm{C}$. At $100 \mathrm{MHz}$ this corresponds to $1.6 \pm 0.1 \mathrm{~Hz}$ (measured at $24^{\circ} \mathrm{C}$ ). The chemical shift difference decreases as the DMCF is diluted in $\mathrm{CCl}_{4}$ and is smaller in the neat liquid. The long range couplings vary to a limited degree

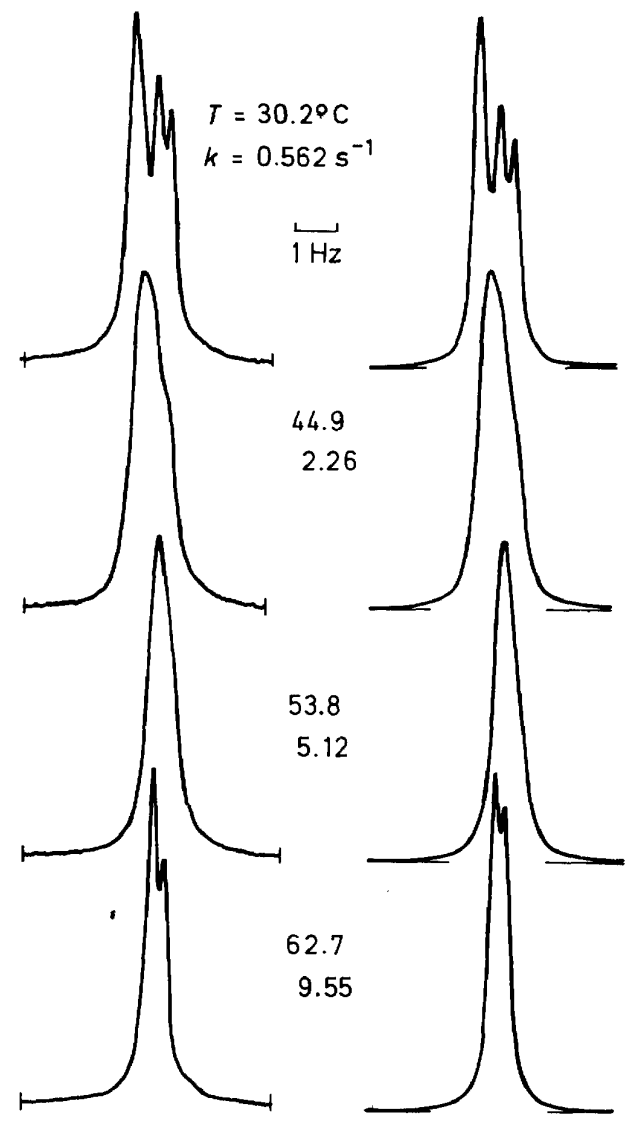

Figure 3. Experimental and computed spectra for the proton magnetic resonance signals in $\mathrm{DMCF}$ at several temperatures. The experimental spectra are presented at the left. 


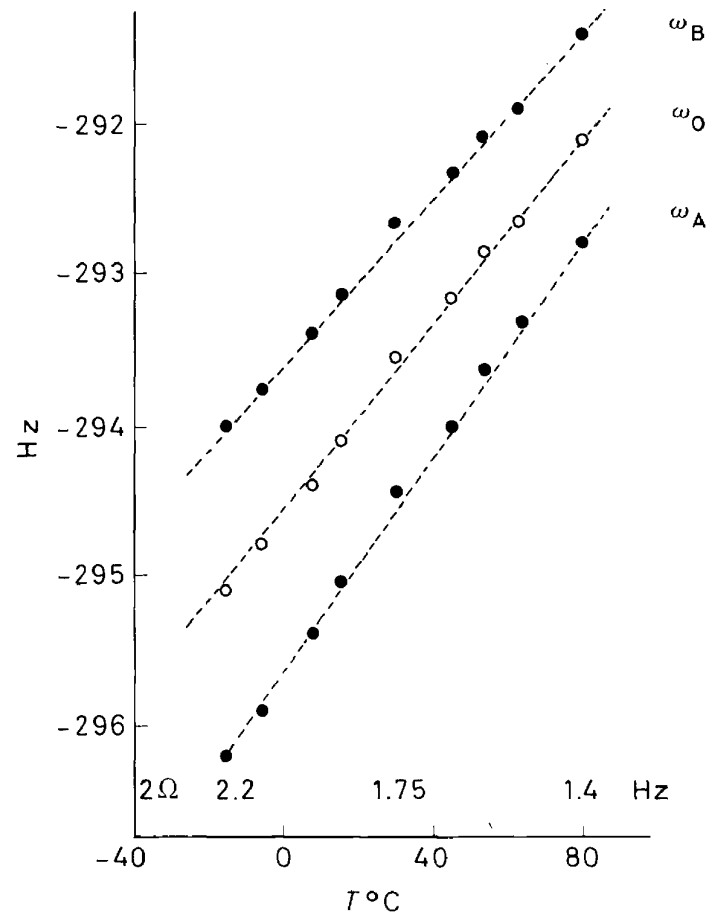

Figure 4. Chemical shift measurements from a TMS reference at $100 \mathrm{MHz}$ for the two methyl proton signals of DMCF $16.5 \%$ in carbon tetrachloride as solvent. The values for $\omega_{A}$ and $\omega_{B}$ were determined from the best line shape fit data at each temperature after approximate values had been assigned. The maximum deviation from a straight line is $0.05 \mathrm{~Hz}$. This illustrates the consistency of the data and the ability to seek exact line positions using line shapes.

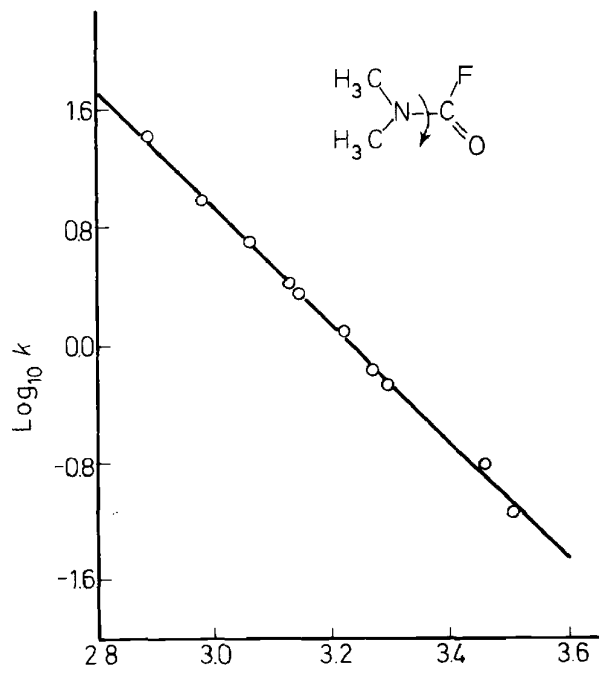

Figure 5. The Arrhenius graph for internal rotation rates about the $\mathrm{C}-\mathrm{N}$ bond in DMCF measured in this work. The best slope was determined by a least squares fit to a straight line. 


\section{MULTI-SITE CHEMICAL EXCHANGE BY NMR}

with concentration but not perceptibly with temperature. For a $7.5 \%$ and 16.5 mole \% solution in $\mathrm{CCl}_{4}, J_{\mathrm{BX}}=0.8 \pm 0.05 \mathrm{~Hz}$ (trans to the fluorine and at higher field) and $J_{\mathrm{AX}}$ (cis $=0.3 \pm 0.05 \mathrm{~Hz}$ while for the neat liquid $J_{\mathrm{BX}}=$ $1.1 \pm 0.05$ and $J_{\mathrm{Ax}}=0.4 \pm 0.05 \mathrm{~Hz}$. The errors on these $J$ values are indicative of the precision, with which line position can be defined in the complete line shape fit.

These unfortunate proton magnetic resonance parameters with which nature endowed DMCF make it by far the most difficult line shape analysis problem yet attempted. At no point in the region of exchange is the total width of the spectrum greater than $3 \mathrm{~Hz}$. It is however, an important molecule in the general amido-type series and an estimate of the barrier by coalescence temperatures is excluded by the nature of the spectrum.

In Figure 3 the experimental spectra are presented at the left and the complete line shape fit spectra are presented on the right as computed by the methods described earlier in this paper. The variation of the chemical shift between the two $\mathrm{N}$-methyl group protons is presented in Figure 4. It is linear over the temperature region studied and varies between $2.2 \mathrm{~Hz}$ at the lower temperatures to $1.4 \mathrm{~Hz}$ at the higher temperatures. No experimental point varies more than $0.05 \mathrm{~Hz}$ from the best straight line as optimized in the complete line shape fit. The Arrhenius graph for this molecule is presented in Figure 5 together with the rate constants and errors for the individual line shape fit data in Table 1. The entropy of activation as calculated in the usual manner ${ }^{22}$ is satisfactorily zero within experimental error for this simple hindered rotation.

Table 1. Temperatures and corresponding rate constants for the internal rotation rates about the $\mathrm{C}-\mathrm{N}$ bond in DMCF. The activation parameters determined from Figure 5 are given at the foot of the table.

\begin{tabular}{|c|c|c|c|c|c|}
\hline \multicolumn{6}{|c|}{ Kinetic data for DMCF, $\left(\mathrm{CH}_{3}\right)_{2} \mathrm{NCOF}$} \\
\hline$T,{ }^{\circ} \mathbf{C}$ & $k, \mathrm{~s}^{-1}$ & error* & $T,{ }^{\circ} \mathrm{C}$ & $k, s^{-1}$ & error* \\
\hline 12.2 & 0.076 & 4.4 & 44.9 & 2.29 & 1.2 \\
\hline 15.6 & 0.159 & 4.2 & 46.5 & 2.57 & 1.3 \\
\hline 30.2 & 0.550 & 3.7 & 53.8 & 5.11 & 1.3 \\
\hline 32.5 & 0.692 & 4.1 & 62.7 & 9.55 & 3.9 \\
\hline 37.6 & 1.99 & 2.2 & 78.2 & 26.3 & 4.6 \\
\hline
\end{tabular}
$E_{a}=18.3 \pm 0.6 \mathrm{kcal} \mathrm{mole}{ }^{-1} ; \log _{10} A=12.9 ; \Delta H^{*}=17.7 \pm 0.6 \mathrm{kcal} \mathrm{mole}{ }^{-1} ; \Delta S^{*}=-1.4 \pm 2.1 \mathrm{cal} \mathrm{deg}^{-1} \mathrm{~mole}^{-1} ; \Delta G^{*}=$
$18.2 \pm 0.6 \mathrm{kcal} \mathrm{mole}{ }^{-1}$

* Percentage error for iterative line shape fit, see text.

The spectrum in the fast exchange limit is an $\mathrm{A}_{6}^{\prime} \mathrm{X}$ grouping with a computed coupling from the line shape data $J_{\mathrm{A}^{\prime} \mathrm{X}}=0.55 \pm 0.05 \mathrm{~Hz}$. This in itself is sufficient to indicate that $J_{\mathrm{AX}}$ and $J_{\mathrm{BX}}$ have the same relative sign. The best fit errors for the same and opposite relative sign assignments differ at $44.9^{\circ} \mathrm{C}$, the lower error, $1.2 \%$, corresponding to the same relative signs and the larger error, $2.0 \%$, for opposite signs.

It is important to question the influence of intermethyl group proton couplings and the influence of proton- ${ }^{14} \mathrm{~N}$ coupling in the line shapes. It is estimated that $J_{\mathrm{NA}}$ and $J_{\mathrm{NB}}$ are of the order $0.7 \mathrm{~Hz}$ but interruption of this 
scalar coupling by rapid $T_{1}$ relaxation of ${ }^{14} \mathrm{~N}$ reduces the effective contribution to the proton line width to zero. Intermethyl coupling is extremely small. certainly less than $0.01 \mathrm{~Hz}$. It is observable in $N, N$-dimethylthiocarbamyl cyanide $\sim 0.1 \mathrm{~Hz}$ and $N, N$-dimethylnitrosamine $\sim 0.1 \mathrm{~Hz}$ but these are extreme cases of high barriers for the $\mathrm{C}-\mathrm{N}$ rotation where the double bond character $\mathrm{C}=\mathrm{N}$ is considerably greater.

The small chemical shift difference in DMCF can be ascribed to similar magnetic susceptibility anisotropies for the $\mathrm{C}=\mathrm{O}$ and $\mathrm{C}-\mathrm{F}$ bonds and approximately equal angles $\mathrm{N}-\mathrm{C}-\mathrm{F}$ and $\mathrm{NC}=\mathrm{O}$.

\section{Dimethyltin dihalide exchange-purity considerations}

The experimental work completed here using chloroform as a solvent, (no matter how highly purified,) indicated an accelerated exchange rate between methyltin halides of the halogen moiety, which changed with time. Chloroform is known to generate impurities on standing; as these catalysts increase in concentration the exchange rate increases. Alcohol which is added as a stabiliser to commercial chloroform is a catalyst itself, but it was carefully removed by distillation and treatment with molecular sieves. In carbon tetrachloride and carbon disulphide, after purification by distillation and storage over molecular sieve, the spectra of dimethyltin diiodide mixed with dichloride were resolved as three relatively sharp peaks at room temperature. In the purest chloroform the best spectra obtainable were three broad peaks at low temperature $\left(-50^{\circ} \mathrm{C}\right)$, which merged below room temperature into two peaks. After considerable experimentation the solvent chosen for the kinetic study was toluene, because it is easy to purify, is nonpolar and has a large liquid temperature range. Dimethyltin dichloride becomes somewhat insoluble below $-50^{\circ} \mathrm{C}$ but this disadvantage is not serious.

\section{Equilibrium measurements for mixtures of dimethyltin dihalides}

In carefully purified dry chloroform, equilibrium measurements can be made in the temperature region $-60^{\circ} \mathrm{C}$ to $+25^{\circ} \mathrm{C}$. A series of determinations for $0.06 \mathrm{M}$ initial concentrations each of dimethyltin dichloride and dimethyltin diiodide give equilibrium quotients from peak areas from 1.97 at $-56^{\circ} \mathrm{C}$ to 2.63 at $30.1^{\circ} \mathrm{C}$. The enthalpy of reaction is approximately 0.35

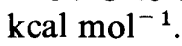

Corresponding measurements for the dibromide and diiodide mixtures were repeated both in chloroform and toluene as solvents. Initial concentrations were $0.06 \mathrm{M}$ each in chloroform and $0.35 \mathrm{M}$ dibromide and $0.15 \mathrm{M}$ diiodide in toluene. Equilibrium quotients varied between 2.70 at $-52^{\circ} \mathrm{C}$ to 3.06 at $-20^{\circ} \mathrm{C}$ in chloroform with an approximate enthalpy of reaction $0.28 \mathrm{kcal} \mathrm{mol}^{-1}$. In toluene, determinations of the equilibrium quotient varied from 2.9 to 4.1 in the range $-30^{\circ} \mathrm{C}$ to $+70^{\circ} \mathrm{C}$. The equilibrium quotient redetermined at several concentrations did not vary within experimental error $( \pm 0.2)$.

A single measurement for the dichloride and dibromide at $-40^{\circ} \mathrm{C}$ with initial concentrations $0.06 \mathrm{M}$ in each component gave a quotient 3.24 for the equilibrium.

It is interesting that the equilibrium does not represent a statistically random distribution of halogen atoms among the dimethyltin moieties. A 


\section{MULTI-SITE CHEMICAL EXCHANGE BY NMR}

random distribution gives an equ 1 brium quotient of 4 . At lower temperatures the symmetric dihalides are favou $e d$.

\section{Preliminary kinetic measurements}

Experimental spectra of a 0.04 molal solution of dimethyltin diiodide and dimethyltin dichloride in toluene are presented for three temperatures in Figure 6. The main peaks can be assigned from low field to high field as the dimethyltin diiodide, the dimethyltin chloroiodide and the dimethyltin

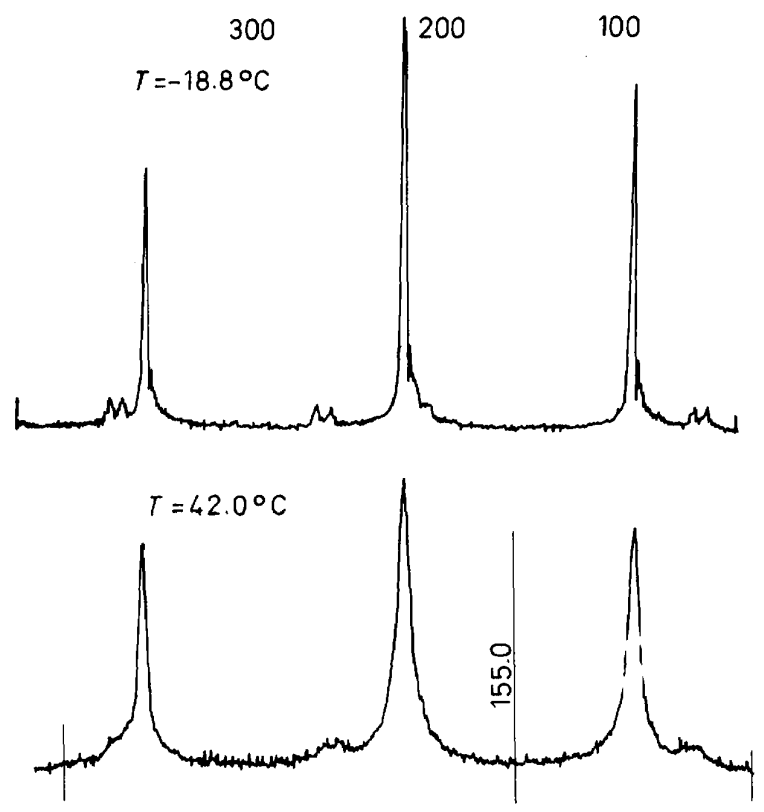

$T=78.8^{\circ} \mathrm{C}$

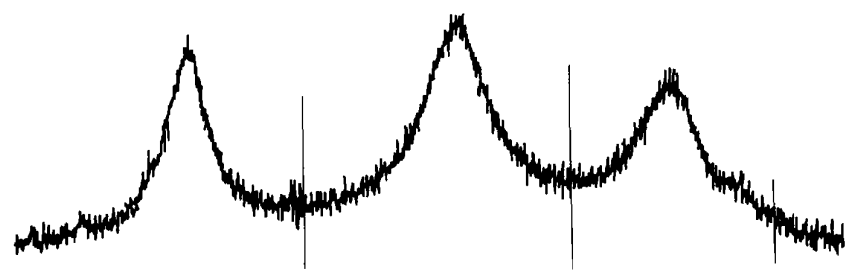

Figure 6. Experimental spectra of a 0.04 molal solution each of dimethyltin diiodide and dimethyltin dichloride in toluene at three temperatures. The low field peak corresponds to the diiodide and the high field signal to the dichloride. The central peak is the mixed dihalide. Tin satellites are clearly resolved for spectra at -18.8 and $42^{\circ} \mathrm{C}$.

dichloride signals respectively. Some of the tin satellite spectra are clearly visible in the spectra at $-18.8^{\circ} \mathrm{C}$ and $42^{\circ} \mathrm{C}$. It is also clear that the exchange rate of the halogens among these three peaks increases with temperature, but not such a rapid increase as found with high barriers of activation in 
amide rotations. The activation energies are obviously considerably smaller.

In Figure 7 are some simulated spectra of this system using various values of the site rate matrix elements, $K_{1}$ and $K_{2}$. It is to be noted that overall features of the experimental spectra are reproduced. The slow exchange limit of $K_{1}=1 \times 10^{-3}$ and $K_{2}=5 \times 10^{-3}$ does not differ from the upper spectrum in Figure 6 where negligible exchange is occurring. The computed spectrum with $K_{1}=2.0, K_{2}=10$ reproduces the second experimental spectrum of Figure 6 at $42^{\circ} \mathrm{C}$.

Detailed fits to determine the chemical rate constants are at present being worked out according to the methods presented earlier at several concentrations of the dimethy!tin halides in toluene.
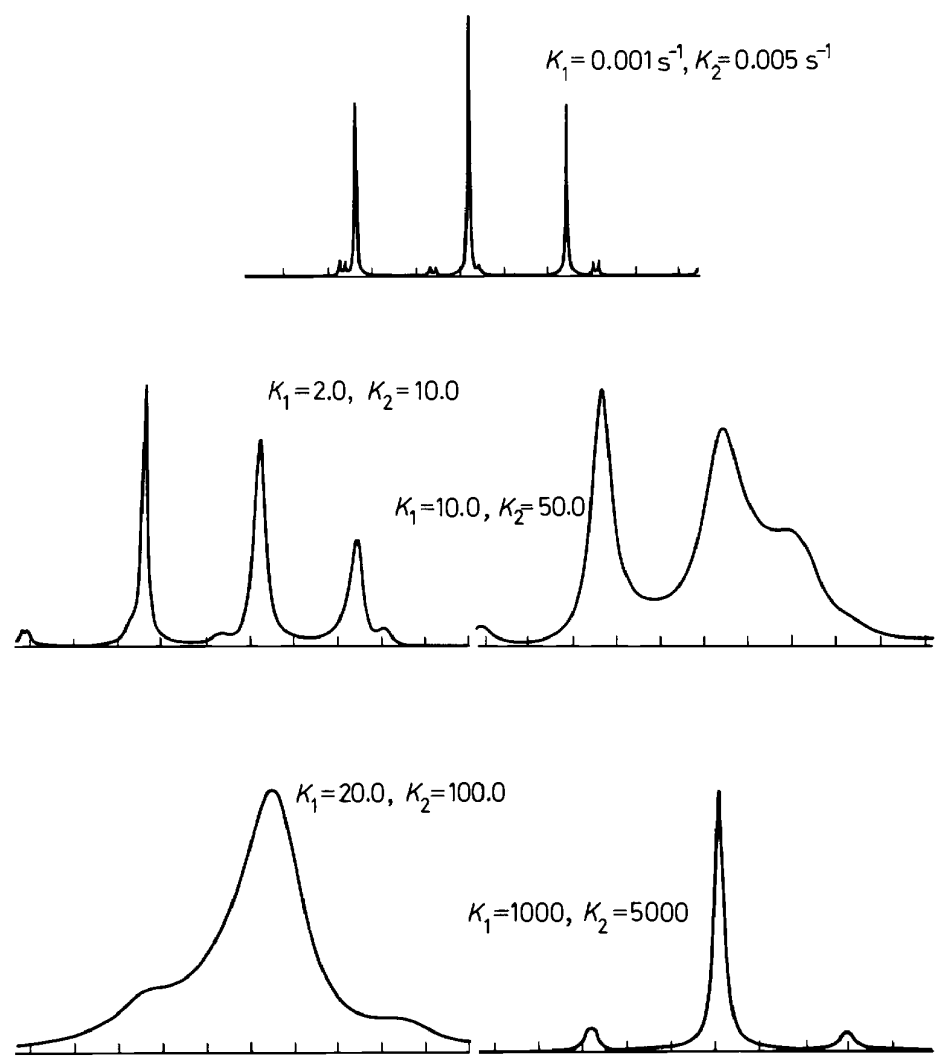

Figure 7. Computed line shapes for the concentrations of methyltin halides designated in Figure 6. The two site rate matrix elements are given for each spectrum.

\section{REFERENCES}

1 H. S. Gutowsky, D. W. McCall and C. P. Slichter, J. Chem. Phys. 21, 279 (1953);

H. S. Gutowsky and C. H. Holm, J. Chem. Phys. 28, 430 (1958).

${ }^{2}$ H. M. McConnell, J. Chem. Phys. 28, 430 (1958).

3 J. Kaplan, J. Chem. Phys. 28, 278 (1958).

4 S. Meiboom, J. Chem. Phys. 34, 375 (1961). 


\section{MULTI-SITE CHEMICAL EXCHANGE BY NMR}

${ }^{5}$ M. Bloom, L. W. Reeves and E. J. Wells, J, Chem. Phys. 42, 1615 (1965).

6 S. Alexander, J. Chem. Phys. 37, 967 (1962).

7 H. S. Gutowsky, R. L. Vold and E. J. Wells, J. Chem. Phys. 43, 4107 (1965).

${ }^{8}$ G. Binsch, J. Am. Chem. Soc. 91, 1304 (1969); Mol. Phys. 15, 469 (1968).

9 L. W. Reeves and K. N. Shaw, Can. J. Chem. 48, 3641 (1970).

${ }^{10}$ L. W. Reeves and K. N. Shaw, Can. J. Chem. In press (1971).

11 L. W. Reeves, R. C. Shaddick and K. N. Shaw, Can. J. Chem. In press (1971).

12 M. T. Rogers and J. C. Woodbury, J. Chem. Phys. 66, 549 (1962).

13 P. T. Inglefield, E. Krakower, L. W. Reeves and R. Stewart, Mol. Phys. 15, 65 (1968).

14 A. Pines and M. Rabinowitz, Tetrahedron Lett. 31, 3529 (1968); J. Am. Chem. Soc. 91, 1585 (1969).

15 H. Kessler, Angew.Chemie., (Intern. Ed.). 9, 219 (1970).

16 J. J. Burke and P. C. Lauterbur, J. Am. Chem. Soc. 83, 326 (1961).

${ }^{17}$ E. V. Van der Berghe, G. P. Van der Kelen and Z. Eeckhaut, Bull. Soc. Chim. Belges. 76, 79 (1967).

18 G. Schrader, British Intelligence Objectives Subcommittee Final Report 714 BIOS London (1945); Z. Arnold, Coll. Czech. Chem. Comm. 24, 760 (1959).

19 A. L. Van Geet, Anal. Chem. 40, 2227 (1968).

${ }^{20}$ F. Bloch, Phys. Rev. 7, 460 (1946).

21 K. I. Dalqvist and S. Forsen, J. Phys. Chem. 69, 4062 (1965).

22 A. Allerhand, H. S. Gutowsky, J. Jonas and R. A. Meinzer, J. Am. Chem. Soc. 44, 3185 (1966). 